\title{
IDENTIFICATION OF DYNAMIC MYOELECTRIC SIGNAL-TO-FORCE MODELS DURING ISOMETRIC LUMBAR MUSCLE CONTRACTIONS
}

\author{
Darryl G. Thelen, Albert B. Schultz, Spilios D. Fassois and James A. Ashton-Miller \\ Biomechanics Research Laboratory, Department of Mechanical Engineering and Applied Mechanics, \\ University of Michigan, Ann Arbor, Michigan, U.S.A.
}

\begin{abstract}
A 14-muscle myoelectric signal (MES)-driven muscle force prediction model of the L3-L4 cross section is developed which includes a dynamic MES-force relationship and allows for cocontraction. Model parameters are estimated from MES and moments data recorded during rapid exertions in trunk flexion, extension, lateral bending and axial twist. Nine young healthy males participated in the experimental testing. The model used in the parameter estimation is of the output error type. Consistent and physically feasible parameter estimates were obtained by normalizing the RMS MES to maximum exertion levels and using nonlinear constrained optimization to minimize a cost function consisting of the trace of the output error covariance matrix. Model performance was evaluated by comparing measured and MES-predicted moments over a series of slow and rapid exertions. Moment prediction errors were on the order of 25,30 and $40 \%$ during attempted trunk flexion-extensions, lateral bends and axial twists, respectively. The model and parameter estimation methods developed provide a means to estimate lumbar muscle and spine loads, as well as to empirically investigate the use and effects of cocontraction during physical task performances.
\end{abstract}

\section{INTRODUCTION}

Quantification of lumbar spine loads during the performance of physical tasks is relevant to improve the understanding of the causes of low back pain. Because spinal loads are not directly measurable in vivo, indirect methods of estimating the internal trunk loads resulting from task performances are needed. Force and moment equilibrium analysis across an imaginary transverse cross section of the lumbar trunk can be used to establish a relationship between the external forces associated with a task and the internal trunk loads (Schultz and Andersson, 1981). However, the mechanical equilibrium equations are typically underdetermined with the number of unknowns (muscle forces, spine loads, ligament tension, abdominal pressure) exceeding the number of equations.

Three alternative methods of solving underdetermined joint equilibrium equations have been utilized. The first method involves the use of simplifying assumptions such that the equilibrium equations become determinate. For example, sagittal moments about the spine have been assumed to be generated by a single-equivalent back muscle (McNeil et al., 1980). This approach allows for direct calculation of the agonistic muscle force from moment equilibrium and spine loads from force equilibrium. While this approach is simple, the conditions under which the assumption of a single-equivalent agonistic muscle

Received in final form 23 September 1993.

Author to whom correspondence should be addressed: Darryl G. Thelen, Dept. of Mechanical Engineering and Applied Mechanics, University of Michigan, 3216 G. G. Brown Building, Ann Arbor, MI 48109-2125, U.S.A. model is valid are limited to physical tasks in which no coactivation of agonistic-antagonistic muscle pairs is present and load-sharing among all agonistic muscles is constant.

A second method of solving underdetermined joint equilibrium equations involves the use of numerical optimization. Muscle forces are predicted that satisfy joint equilibrium and minimize an objective function which is designed to reflect the assumed muscular recruitment strategy (Hughes, 1991; Schultz et al., 1983). Although good correlations have been found between model-predicted muscle forces and lumbar myoelectric signals (MES) during light to moderate sagittal loadings (Schultz et al., 1983), substantially weaker MES-force correlations are observed when tasks involve heavy exertions (Schultz et al., 1987) and three-dimensional loadings (Hughes, 1991). The major drawbacks of this approach are that it is difficult to account for subject and task-dependent variations in muscle recruitment, and it commonly does not predict cocontraction which becomes substantial during three-dimensional loadings of the trunk (McGill, 1991; Pope et al., 1986).

The third approach to estimating internal joint loadings is to predict muscle contraction forces from MES. A major advantage of this approach is that empirical measures (MES) are used directly as quantitative indicators of muscle recruitment. Thus, interindividual and task-to-task variabilities in muscle recruitment can be accounted for, and the effects and use of cocontraction can be quantified. While MES have been used extensively to predict joint torques in the extremities (Bobet and Norman, 1990; Hof, 1984; Olney and Winter, 1985; Redfern, 1988), predicting lumbar muscle forces from MES presents a formidable task due to the large number of trunk muscles, 
their capacity to generate substantial three-dimensional torques as well as the complexities of the MES-force relationship. McGill and Norman (1986) developed the earliest MES-driven lumbar muscle force prediction model using it to estimate lumbar trunk loads during dynamic, sagittal lifting tasks. Marras and Sommerich (1991) estimated lumbar muscle forces from MES during isokinetic trunk extensions. While muscle length and velocity affects were accounted for in these models, the MES-force modeling was based on a priori assumptions of the MES-stress relationship and only sagittal moments were considered. An attempt by McGill (1991) to use MES to predict muscle forces and moments during axial twist exertions resulted in poor agreement with measured moments. Hughes (1991) used principal components regression to calibrate individualized MES-force models during asymmetric static tasks but included only six muscles and did not consider rapid exertions or twisting moments.

The objective of the present study was to develop, calibrate and evaluate an isometric lumbar MESto-force prediction model which differs from previous models in the following respects: (1) MES from 14lumbar muscles were accounted for, (2) dynamic MES-force models are estimated from experimental data, (3) model calibration considered three-dimensional loading conditions, (4) model performance was evaluated over tasks involving slow and rapid threedimensional exertions.

\section{METHODS}

Nine healthy male subjects participated in this study. Their ages ranged from 21 to 31 yr with a mean of $24.1 \mathrm{yr}$, heights ranged from 168.0 to $190.5 \mathrm{~cm}$ with a mean of $175.8 \mathrm{~cm}$ and body weights ranged from 61.0 to $83.9 \mathrm{~kg}$ with a mean of $73.2 \mathrm{~kg}$. All tasks were performed in an upright position in a test frame which resisted lower body motion [Fig. 1(a)].

Each subject performed a total of 22 tasks which included six maximum voluntary exertions (MVE), four calibration tasks and 12 validation tasks. The MVE tasks were performed for $2 \mathrm{~s}$ intervals with at least 1 min rest between tasks and consisted of maximally attempted: (1) trunk flexion; (2) trunk extension; (3) right lateral bend (rlb); (4) left lateral bend (llb); (5) counter-clockwise (ccw) twist; (6) clockwise (cw) twist. During the calibration tasks, restraint pads were placed anteriorly, posteriorly and laterally to the upper body at shoulder level. In the first calibration task, the subject was asked to sequentially attempt trunk flexion, extension, right lateral bend, left lateral bend, ccw twist and cw twist. A second calibration task was performed similarly but without attempted axial twist. Each calibration task was repeated once. Rapid exertions to approximately two thirds of MVE were performed with a metronome to guide the rate of sequencing through the exertion directions. Eighteen and $12 \mathrm{~s}$ of data were recorded during the calibration tasks with and without axial twist, respectively.

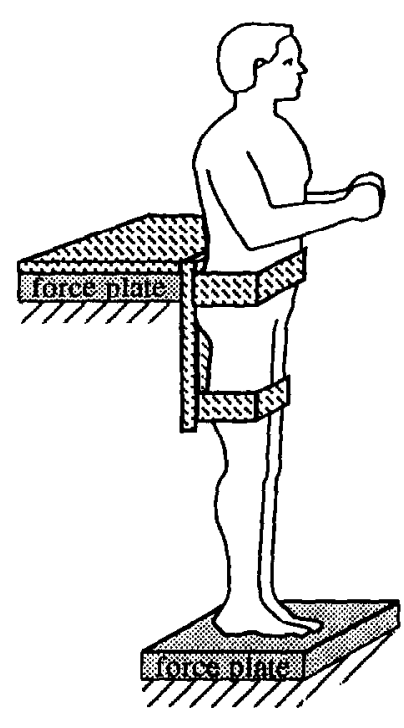

(a)

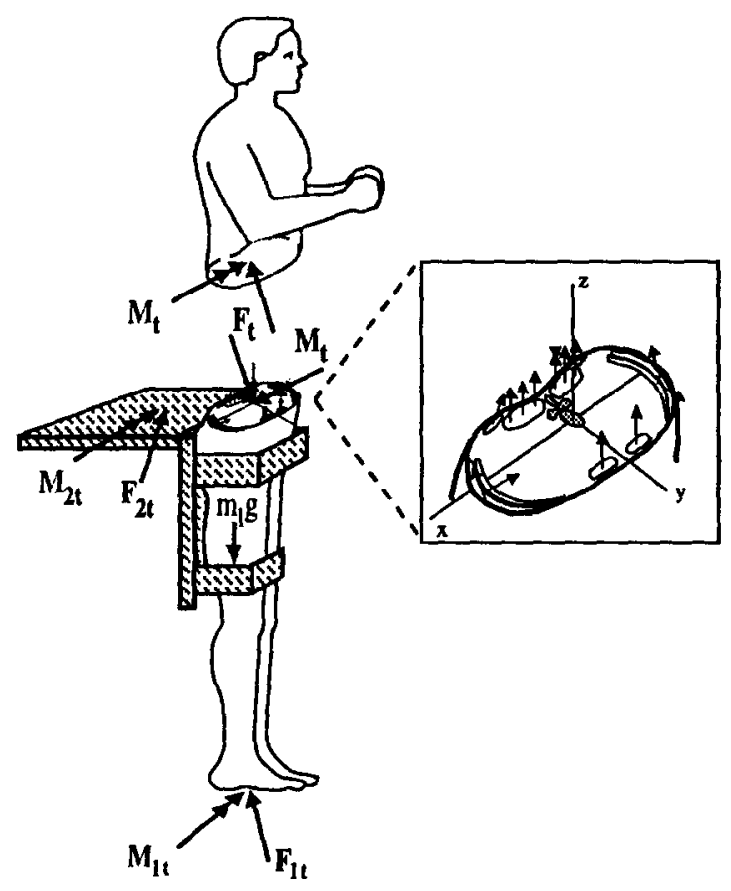

(b)

Fig. 1. (a) Schematic representation of the experimental set-up. Padded restraints were placed around the upper body during attempted exertions. (b) Free body diagram used to calculate the net forces and moments at the L3-L4 level of the trunk. Moments about the lumbar spine were assumed to be generated by the 14-muscles included in the model of the transverse cross section of the lumbar trunk. 
The set of validation tasks included four attempted flexion-extensions, four attempted lateral bends and four attempted axial twists. Each set of four tasks consisted of a slow cyclic bi-directional exertion (6 cycles per minute), a fast cyclic bi-directional exertion (40 cycles per minute) and two single direction pulse exertions ( $3 \mathrm{~s}$ duration). Upper body restraints were placed to minimize exertions in directions other than those prescribed (Thelen, 1992). All validation tasks were performed to approximately $60 \%$ of MVE levels with an oscilloscope display of force plate reactions used as indicators of effort level. In all tasks, the subject was first given sufficient practice to master tracking a desired reaction force. The feedback signal was turned off during actual data collection to minimize constant corrective actions associated with force tracking.

Two force plates (AMTI) were used to measure foot-floor and pelvis-support reaction forces (Fig. 1). The relative position of the two force plate origins was measured to the nearest millimeter using a plumb bob and rule. Force plate signals were amplified with a gain of 2000 and sampled by a 12 bit A/D board at $100 \mathrm{~Hz}$. Trunk position data were measured using an optoelectronic motion measurement system (WATSMART; Northern Digital Inc.). Two cameras were located approximately $3 \mathrm{~m}$ behind the subject. Markers were positioned on the skin overlying the posterior spinous processes of the second and fourth lumbar vertebral bodies, and at the origin of the pelvis support force plate. Position data were collected at $100 \mathrm{~Hz}$. Force plate and kinematic data were subsequently digitally low-pass filtered using a secondorder Butterworth filter with a cutoff frequency of $5 \mathrm{~Hz}$; forward and backward passes were used to remove phase shift. The position of the L3-L4 disc centroid was estimated relative to the back markers using the regression equation reported by Tracy et al. (1989).

The experimental set-up resisted any motions of the lower body allowing the moments $\left(\mathbf{M}_{t}\right)$ and forces $\left(\mathbf{F}_{t}\right)$ necessary to equilibriate the upper body at any time step to be calculated using a static analysis of the lower body [Fig. 1(b)]:

$$
\begin{aligned}
& \mathbf{M}_{t}=\sum_{i=1}^{2} \mathbf{d}_{i t} \times \mathbf{F}_{i t}+\mathbf{M}_{i t}, \\
& \mathbf{F}_{t}=\sum_{i=1}^{2} \mathbf{F}_{i t}-m_{\ell} g,
\end{aligned}
$$

where $d_{i t}$ is the vector from the center of the L3-L4 disk to the origin of force plate $i$ at time step $t, m_{\ell}$ is the mass of the lower body, $g$ is the acceleration of gravity, and $\mathbf{F}_{\text {it }}$ and $\mathbf{M}_{i t}$ are the force and moment vectors recorded by force plate $i$ at time step $t$.

MES were recorded using seven recessed bipolar surface electrodes on each side of the trunk oriented parallel to the underlying fiber direction. Electrodes consisted of two $10 \mathrm{~mm}$ diameter silver disks spaced $20 \mathrm{~mm}$ center-to-center. Three pairs of electrodes were placed bilaterally over the erector spinae (ES) muscles at the L3-L4 level: 2,4 , and $6 \mathrm{~cm}$ lateral to the midline. These were approximately over the multifidus (ESM), longissimus (ESL) and iliocostalis (ESI) columns of the lumbar ES muscle. An electrode pair was placed over the latissimus dorsi (LD) muscle at the T10 level, two-thirds of the distance form the midline to the lateral aspect of the body. Six abdominal electrodes were placed bilaterally over the rectus abdominis (RA), internal oblique (IO) and external oblique (EO) muscles. RA electrodes were placed $3 \mathrm{~cm}$ lateral to the midline at the level of the umbilicus. IO electrodes were placed $2 \mathrm{~cm}$ inferior and $3 \mathrm{~cm}$ medial to the anterior superior iliac spine. EO electrodes were placed at the lateral aspect of the body at the level of the umbilicus.

Raw myoelectric signals were differentially amplified to volt levels and digitized at $1500 \mathrm{~Hz}$ through a 16 channel 12 bit A/D board. The signal from each electrode was checked visually on a screen before and frequently during the testing. The myoelectric signals were digitally high-pass filtered with a second-order high-pass Butterworth filter with a cutoff frequency of $30 \mathrm{~Hz}$; a forward and backward pass were used to remove phase shift. This filter was used to attenuate the noise on the MES due to heartbeat and movement artifacts. The RMS MES were obtained by squaring the MES data sequence, digitally convolving it with a center average rectangular window and taking the square root of this sequence. The window length, $h$, was set equal to 75 points or $50 \mathrm{~ms}$ resulting in the squared myoelectric signals being low-pass filtered at a cutoff frequency of $8.9 \mathrm{~Hz}$. All RMS MES were normalized to the maximum mean RMS MES recorded for that muscle over the series of six MVE tasks.

\section{MODEL DEVELOPMENT}

A model relating the normalized RMS MES to the measured three-dimensional moments about the spine was developed. The following assumptions were made and will be reviewed in more detail in the discussion:

(1) Internal moments about the spine are produced only by active muscular contraction in an upright standing position.

(2) Muscle forces at the L.3-L.4 cross section can be represented by a vector with a constant direction acting at the muscle centroid.

(3) The muscles included in the model are representative of all the lumbar muscles used by the subjects during task performances.

(4) Muscle geometry data taken from the literature accurately represent the muscle geometries of the subjects that participated in this study.

(5) Muscle length changes during the quasi-isometric tasks were negligible.

(6) The isometric RMS MES-force relationship can be described by linear dynamical equations with common temporal characteristics for all muscles. 
The dynamic relationship between the RMS MES and isometric muscle stress was represented by a linear discrete time dynamic model:

$$
\sigma_{i t}+a_{1} \sigma_{i t-1}+\cdots+a_{m} \sigma_{i t-m}=b_{i} u_{i t-k},
$$

where $u_{i t}$ and $\sigma_{i z}$ are the normalized RMS MES and MES-predicted muscle stress of the $i$ th muscle at time step $t, k$ is a pure time delay between the onset of MES and the onset of muscle stress, and $\left\{a_{1} \ldots a_{m}, b_{i}\right\}$ are adjustable parameters. In more compact notation, the MES-stress model can be written in transfer function form as:

$$
\sigma_{i t}=\frac{b_{i}}{A(B)} u_{i t-k},
$$

where $B$ represents the backshift operator \{i.e. $\left.B \cdot u_{i t}=u_{i t-1}\right\}$ and $A(B)$ is a polynomial in $B$ of order $m:$

$$
A(B)=1+a_{1} B+a_{2} B^{2}+\cdots+a_{m} B^{m} .
$$

A 14 muscle geometric representation of the L3-L4 transverse cross section was used to relate the muscle stresses to the net moments about the spine (Table 1). The centroid locations and cross-sectional areas for the RA, abdominal obliques and ES muscles were taken from Tracy et al. (1989) who used magnetic resonance imaging to take these measurements. Muscle lines of action (LOA) for the RA, IO, EO, ESL, ESI and ESM muscles were based on a LOA digitization study by Dumas et al. (1988) and an anatomical study by Macintosh and Bogduk (1986). The centroid location and LOA of the LD was estimated by assuming equal stress for the parts of this muscle which act directly on the iliac spine and on the lumbar spine via the lumbodorsal fascia (Bogduk and Macintosh, 1984). The erector spinae CSA was divided into the ESM, ESL and ESI columns using the ratios given in Schultz et al. (1983). The abdominal oblique CSA was divided into the IO and EO muscles using the ratio found by Kumar (1988). Physiological cross sectional arcas (PCSA) were obtained by multiplying the anatomic CSA by the absolute value of the $z$ component of the muscle LOA unit vector. The LD PCSA acting through the L3-L4 cross section was estimated as $50 \%$ of the total muscle PCSA (Veeger et al., 1991). Muscle CSA and centroid locations were not scaled for each subject since anthropometric measures have not adequately explained a significant percentage of inter-subject variations in lumbar muscle geometries (Kumar, 1988; Tracy et al., 1989).

The moment about the L3-L4 disk centroid generated by the $i$ th muscle contracting with a stress of one $\mathrm{N} \mathrm{cm}{ }^{-2}$ is dependent only on muscle geometry and is given by:

$$
\mathbf{m}_{i}=p_{i}\left(\mathbf{r}_{i} \times \mathbf{f}_{i}\right),
$$

where $p_{i}$ is the PCSA of the ith muscle, $\mathbf{r}_{i}$ is a vector from the center of the L3-L4 disk to the centroid of the $i$ th muscle and $f_{i}$ is a unit vector fixed along the LOA of the $i$ th muscle. The net MES-predicted moments are given by summing the moments developed by each of the individual muscles:

$$
\hat{\mathbf{M}}_{t}=\sum_{i=1}^{n} \frac{b_{i}}{A(B)} u_{i t-k} \mathbf{m}_{i}
$$

with $n$ representing the total number of muscles.

\begin{tabular}{|c|c|c|c|c|c|c|c|}
\hline \multirow[b]{2}{*}{ Muscle } & \multirow[b]{2}{*}{ Index } & \multirow{2}{*}{$\begin{array}{c}\text { PSCA } \\
\left(\mathrm{cm}^{2}\right) \\
p_{i}\end{array}$} & \multicolumn{2}{|c|}{$\begin{array}{l}\text { Centroid location } \\
\text { (m) }\end{array}$} & \multicolumn{3}{|c|}{ Unit vector along LOA } \\
\hline & & & $r_{x}$ & $r_{y}$ & $f_{x}$ & $f_{y}$ & $f_{z}$ \\
\hline RAR & 1 & 6.60 & 0.034 & 0.080 & -0.028 & 0.016 & -0.999 \\
\hline RAL & 2 & 6.60 & -0.034 & 0.080 & 0.028 & 0.016 & -0.999 \\
\hline IOR & 3 & 13.13 & 0.114 & 0.010 & 0.134 & -0.574 & -0.808 \\
\hline IOL & 4 & 13.13 & -0.114 & 0.010 & -0.134 & -0.574 & -0.808 \\
\hline EOR & 5 & 16.40 & 0.126 & 0.011 & -0.376 & 0.322 & -0.870 \\
\hline EOL & 6 & 16.40 & -0.126 & 0.011 & 0.376 & 0.322 & -0.870 \\
\hline ESMR & 7 & 5.20 & 0.018 & -0.061 & 0.309 & 0.000 & -0.951 \\
\hline ESML & 8 & 5.20 & -0.018 & -0.061 & 0.309 & 0.000 & -0.951 \\
\hline ESLR & 9 & 10.30 & 0.039 & -0.060 & -0.134 & 0.005 & -0.991 \\
\hline ESLL & 10 & 10.30 & -0.039 & -0.060 & 0.134 & 0.005 & -0.991 \\
\hline ESIR & 11 & 9.96 & 0.059 & -0.054 & -0.281 & -0.052 & -0.958 \\
\hline ESIL & 12 & 9.96 & -0.059 & -0.054 & 0.281 & -0.052 & -0.958 \\
\hline LDR & 13 & 4.30 & 0.035 & -0.080 & -0.890 & -0.137 & -0.430 \\
\hline LDL & 14 & 4.30 & -0.035 & -0.080 & 0.890 & -0.137 & -0.430 \\
\hline
\end{tabular}

The relationship between the measured moments and normalized RMS MES was expressed in an out-

Table 1. Trunk muscle physiological cross sectional areas (PCSA), centroid locations and lines of action (LOA) for fourteen lumbar muscles at the L3-L4 level of the trunk

Orientation: The $x$-axis is directed right laterally, the $y$-axis is directed anteriorly and the $z$-axis is directed superiorly relative to the disc centroid.

Notation: RA-rectus abdominis, IO-internal oblique, EO-external oblique, ESMerector spinae multifidus, ESL-erector spinae longissimus, ESI-erector spinae iliocostalis, LD-latissimus dorsi, $\sim$ R-right, $\sim$ L-left. 
put error (OE) form:

$$
\mathbf{M}_{t}=\sum_{i=1}^{n} \frac{b_{i}}{A(B)} u_{i t-k} \mathbf{m}_{i}+\mathbf{e}_{t}(\mathbf{X})
$$

where $e_{1}(X)$ is the difference between the measured and MES-predicted moments at time step $t$. The explicit dependence of this prediction error, $\mathbf{e}_{\mathbf{t}}(\mathbf{X})$, on the adjustable parameters is denoted by the vector $\mathbf{X}$ :

$$
\mathbf{X}=\left[a_{1} a_{2} \cdots a_{m} b_{1} b_{2} \cdots b_{n}\right]^{\mathbf{T}}
$$

with $\mathrm{T}$ indicating vector-matrix transposition.

Each input to the OE model consists of a timevarying scalar, $u_{i t}$, weighted by a constant vector, $\mathbf{m}_{i}$. The cross correlation, at zero lag, between any two such inputs is given by:

$$
R_{i j}=\rho_{i j} \cos \phi_{i j},
$$

where $\rho_{i j}$ is the normalized linear correlation of the RMS MES of the $i$ th and $j$ th muscles and $\phi_{i j}$ is the angle between the moment vectors $\mathbf{m}_{i}$ and $\mathbf{m}_{j}$. Some of the RMS MES and model input cross correlations during the calibration tasks were found to be substantial (Table 2). For example, pairs of ES muscles on the same side had a linear cross correlation exceeding 0.90. This high degree of correlation among model inputs, referred to as multicolinearity, has been shown to result in highly inconsistent parameter estimates in the sense that estimates can vary considerably over repeated calibration task performances (Hughes, 1991).

Three different candidate models (I, II, and III) were identified and compared. In Model I, no special procedures were used to address the potential adverse effects of input multicolinearity on parameter estimation. Model II is identical to Model I in structure, but a principal component analysis approach (Neter $e t$ al., 1990) was used in the parameter estimation to circumvent 'inconsistent' (nonrepeatable) estimates due to numerical difficulties originating from multicolinearity. In this case, the model inputs were first linearly transformed such that the transformed inputs, termed the principal components, were minimally correlated. Model parameters were then estimated using the minimum number of principal components which accounted for $95 \%$ of the input variance (for details, see Appendix A).

In Model III, the normalized RMS MES-stress gains, $b_{i}$, were constrained to be the same for muscles whose activities were most highly correlated. Justification for these constraints are based on the normalization of RMS MES to MVE levels, with the assumption being that the maximum MVE stresses developed by muscles with correlated activities are the same. The three erector spinae muscles on either side of the body, which correlated with $R_{i j}>0.90$, were constrained to have the same MES-stress gains. The right and left RA were constrained to have the same MES-stress gains based on a correlation of $R_{i j}>0.58$ and assumed lateral symmetry. The IO, EO and LD on each side of the body, which linearly correlated with $\rho_{i j}$ exceeding 0.47 , were also constrained to have the same MES-stress gains. Model III was given by expression (8) with the following equality constraints on the MES-stress gains:

$$
\begin{aligned}
& b_{1}=b_{2}, \quad b_{3}=b_{5}=b_{13}, \quad b_{4}=b_{6}=b_{14} \\
& b_{7}=b_{9}=b_{11}, \quad b_{8}=b_{10}=b_{12},
\end{aligned}
$$

where the muscle indices are given in Table 1.

Parameter estimation was based on the minimization of one-half of the trace of the sample covariance matrix of the output error vector:

$$
\mathbf{V}=\operatorname{Trace}\left[\frac{1}{2 N} \sum_{t=1}^{N} \mathbf{e}_{t}(\mathbf{X}) \mathbf{e}_{t}^{\mathrm{T}}(\mathbf{X})\right],
$$

where $N$ is the number of data points available from the calibration task and $e_{t}(X)$ is the output error vector defined by expression (8) for Models I and III and expression (A2) for model II. This OE approach was selected because it is known to allow for the statistically consistent (asymptotically unbiased) estimation of the input-output relationship including any particular form of noise dynamics (Ljung, 1987). The minimization of the cost function given by expression (12) is a nonlinear optimization problem which was solved using a Gauss-Newton optimization technique (see Appendix B).

The error between the measured and MES-predicted moments was quantified by a coefficient of variability (CV), defined as the RMS magnitude of the prediction error normalized by the RMS magnitude of the net moment:

$$
C V=\frac{\sqrt{\sum_{t=1}^{N} \overline{\mathbf{e}_{t}^{\mathrm{T}}(\mathbf{X}) \mathbf{e}_{t}(\mathbf{X})}}}{\sqrt{\sum_{t=1}^{N} \mathbf{M}_{t}^{\mathrm{T}} \mathbf{M}_{t}}} .
$$

Table 2. The maximum mean (S.D.) normalized cross correlations, $\rho_{i j}$, of the RMS MES at zero lag during the calibration tasks. The corresponding cross-correlations of the OE model inputs, $R_{i j}$, include muscle geometry effects. The high degree of correlations among model inputs, referred to as multicolinearity, contributes to difficulties in obtaining repeatable estimates of model parameters

\begin{tabular}{llllllll}
\hline Calibration task & & RAR-RAL & IOR-EOR & ESMR-ESLR & ESLR-ESIR & LDR-ESIR & LDR-IOR \\
\hline w/o twist & $\rho_{i j}$ & $0.87(0.69)$ & $0.71(0.13)$ & $0.97(0.02)$ & $0.92(0.11)$ & $0.37(0.21)$ & $0.28(0.18)$ \\
& $R_{i j}$ & $0.60(0.06)$ & $0.71(0.13)$ & $0.93(0.02)$ & $0.89(0.11)$ & $0.34(0.19)$ & $0.09(0.06)$ \\
w/ twist & $\rho_{i j}$ & $0.84(0.11)$ & $0.62(0.13)$ & $0.84(0.04)$ & $0.90(0.03)$ & $0.38(0.20)$ & $0.47(0.17)$ \\
& $R_{i j}$ & $0.58(0.08)$ & $0.33(0.07)$ & $0.96(0.04)$ & $0.94(0.03)$ & $0.23(0.12)$ & $0.30(0.11)$ \\
\hline
\end{tabular}


Similarly, coefficients of variability about each of the three axes are defined as the RMS magnitude of the scalar error normalized by the RMS magnitude of the net moment.

The candidate models were evaluated based on three criteria. First, model repeatability was quantified by the percent variability in parametcr estimates across repeated trials of the calibration task. Secondly, the physical feasibility of the MES-predicted muscular stresses was evaluated. Predicted muscle stresses must be positive since muscles can act only in contraction and MVE muscle stresses must be within estimated maximal stress levels of $35-100 \mathrm{~N} \mathrm{~cm}^{-2}$ (McGill, 1991; Schultz et al., 1983). Thirdly, the candidate models must be able to predict triaxial moments accurately during tasks other than the specific task for which the model was originally estimated. The three candidate models were identified for a first order $A(B)$ model with no pure time delay and then compared. Only results obtained when using the calibration task which included axial moment development is presented. The effects of not considering axial twisting moments in the calibration is noted in the discussion.

\section{RESULTS}

The mean peak moments across all subjects during the calibration task which included twists were 65.6 (S.D. $=28.8$ ) $\mathrm{N} \mathrm{m}$ in attempted flexion, 95.4 (S.D. $=37.2) \mathrm{Nm}$ in attempted extension, 80.7 $($ S.D. $=27.5) \mathrm{Nm}$ in attempted lateral bending and 40.5 (S.D. $=19.6) \mathrm{Nm}$ in attempted twist (Fig. 2). Maximal mean RMS MES across the MVE tasks ranged from 23.8 to $517.3 \mu \mathrm{V}$.

The variability of parameter estimates across repeated performances of the calibration were model dependent. The first-order characteristic polynomial parameter, $a_{1}$, was consistently estimated (variability $<0.6 \%$ ) using all three candidate models (Table 3). However, the estimated MES-stress gains, $b_{i}$, were substantially variable across calibration task repeats when Models I and II were used in the parameter estimation. The average variability of the MES-stress gains ranged from 21 to $90 \%$ for Model I and from 18 to $91 \%$ for Model II. Mean MES-stress gain variabilities estimated using Model III were substantially lower ranging from 11 to $16 \%$. Negative MES-stress gains, $b_{i}$, correspond to predictions of unattainable negative muscle forces and were obtained for some muscles using Models I and II. Strictly positive MESstress gains were obtained using Model III. Based on this finding and the lowest parameter variability among the three models, Model III was judged better suited than Models I and II to be used in the parameter estimation.

The effects of higher-order models and the inclusion of purc time delays (from 0 to $30 \mathrm{~ms}$ ) on model estimation were investigated using Model III. Of the estimated models, the magnitude of the calibration task model prediction errors were minimized using a second-order model with no pure time delay for each of the subjects (Fig. 3). A dispersion analysis (Fassois and Lee, 1993) demonstrated that the contributions of the second modes to the total output signal energy was substantial (Table 4). The estimated second-order MES-stress models were low-pass systems that werc overdamped for eight of nine subjects and slightly underdamped for one subject (Table 4). The halfpower cutoff frequencies ranged from 0.90 to $1.83 \mathrm{~Hz}$ with a mean of $1.26 \mathrm{~Hz}$ (Fig. 4). The equivalent continuous time poles of these transfer functions were -8.52 (S.D. $=1.83$ ) and -36.59 (S.D. $=18.93$ ) which correspond to time constants of 122 (S.D. $=28$ ) and 34 $(\mathrm{S} . \mathrm{D} .=16) \mathrm{ms}$. For the underdamped model, the natural frequency, damping ratio and exponential envelope time constant were $2.07 \mathrm{~Hz}, 0.39$ and $195 \mathrm{~ms}$. The average MES-predicted MVE stresses, across all nine subjects, for Model III ranged from 37.5 (S.D. $=11.4) \mathrm{N} \mathrm{cm}^{-2}$ for IO, EO and LD muscles to 76.6 (S.D. $=47.8) \mathrm{N} \mathrm{cm}^{-2}$ for the RA muscles (Table 5).

The measured and MES-predicted primary moments were highly correlated during both slow and rapid exertions in trunk flexion, extension, lateral bending and axial twist (Fig. 5). The mean sagittal plane $C V$, expressed as a percentage, ranged from $21 \%$ during pulse attempted extension to $29 \%$ during pulse attempted flexion (Table 6). Cyclic task rate had little effect on the sagittal plane moment prediction errors with the average error being $31 \%$ at the $6 \mathrm{cpm}$ rate and $30 \%$ at the $40 \mathrm{cpm}$ rate. Frontal plane moment errors, expressed as the mean $C V$ across subjects, ranged from 30 to $34 \%$ across the attempted lateral bending tasks. Mean transverse plane moment errors during attempted axial twists ranged from 37 to $44 \%$ but demonstrated large intersubject variability.

\section{DISCUSSION}

The objective of the present study was to identify dynamic MES-driven lumbar muscle force prediction models from experimental data. MES from 14 lumbar muscles were accounted for and time varying, isometric triaxial loadings of the trunk were considered both in the model estimation and performance evaluation.

Various assumptions (see methods) were used in the model development which should be justified and the implications understood. The lumbar muscles at an L3-L4 cross section were assumed to act a constant location with a time-invariant LOA (Schultz and Andersson, 1981). This approximation is probably weakest for the IO and EO muscles where the muscle fibers undergo systematic and substantial fiber direction changes along their lengths, but were represented simply by single LOA. More detailed modeling of this muscle would involve dividing the IO and EO muscles into a number of segments. However, this would require that reliable MES be obtainable for each segment, which is difficult due to the physical 

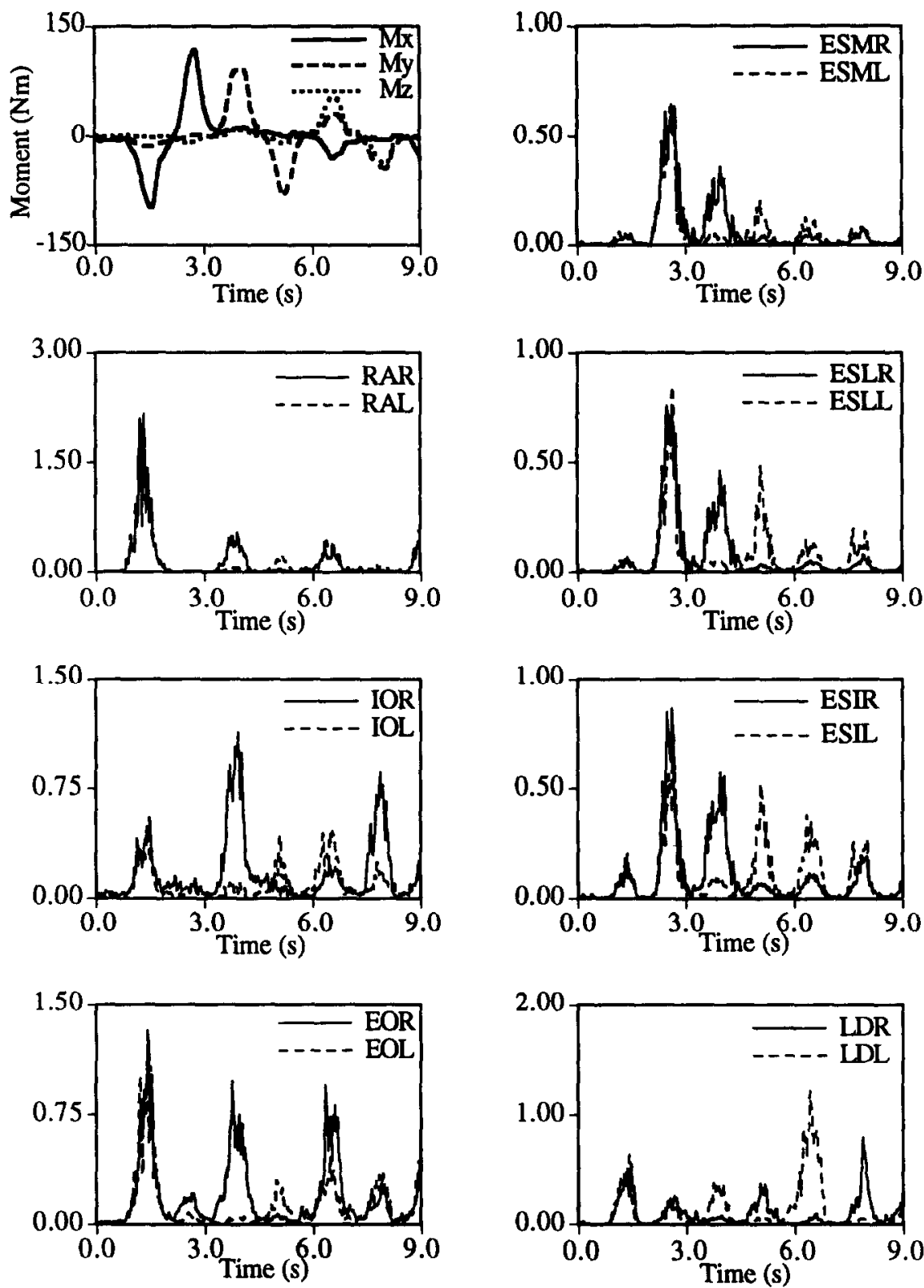

Fig. 2. The moments and normalized RMS MES during nine seconds of a calibration task. Distinct bursts of individual muscle activities are associated with the various exertion directions.

overlapping of the $\mathrm{IO}$ and $\mathrm{EO}$ muscles. We believe that improved monitoring of the IO and EO activities, and modeling of the LOA are crucial to improve load predictions during tasks involving substantial lateral bending and twisting moments. The deep muscles of the trunk, psoas and quadratus lumborum, were excluded because it was not possible to record activities from these muscles using surface electrodes. The psoas muscle is believed to be most active in generating frontal moments (Ladin et al., 1989) which may partially explain explain the underprediction of frontal moments during attempted lateral bends (e.g., see Fig. 5).

A linear discrete time transfer function (Bobet and Norman, 1990; Olney and Winter, 1985) was assumed adequate to represent the rms MES-force relationship. While nonlinear dynamic aspects of the MES-force relationship have been modeled by processing rising and falling MES differently (Hof and Van den Berg, 1984; Redfern, 1988), a linear dynamic model was used because the properties of the resulting identification approach are well understood, techniques for model order selection are available (Ljung, 1987) and estimated models can be related to corresponding continuous time models; something that is difficult within a nonlinear setting. While the phase delay between MES and force may be different for abdominal and back muscles (Thelen et al., 1993), the characteristic polynomial was assumed constant for 
Table 3. Mean (S.D.) parameters for the estimated first-order models of the RMS MES-stress relationship. Also given are the mean percent variation (\% var) of the model parameters over repeated trials of the calibration task. Parameter estimates obtained using Model III were less variables than those obtained using Models I and II

\begin{tabular}{ccccccccc}
\hline & & \multicolumn{7}{c}{$b_{i}$} \\
\cline { 3 - 9 } Model & $a_{1}$ & RAR & IOR & EOR & ESMR & ESLR & ESIR & LDR \\
\hline I mean & -0.936 & 3.3 & 0.9 & 4.2 & 2.4 & 8.5 & -1.5 & 4.2 \\
(S.D.) & $(0.009)$ & $(4.6)$ & $(2.0)$ & $(1.8)$ & $(29.8)$ & $(27.7)$ & $(14.7)$ & $(4.9)$ \\
\% var & 0.510 & 89.4 & 49.5 & 20.7 & 53.8 & 71.5 & 72.0 & 61.3 \\
II mean & -0.934 & 5.4 & 0.4 & 3.6 & 2.9 & 3.1 & 3.0 & 3.3 \\
(S.D.) & $(0.009)$ & $(3.8)$ & $(2.1)$ & $(1.6)$ & $(3.9)$ & $(1.3)$ & $(2.5)$ & $(4.2)$ \\
\% var & 0.590 & 24.4 & 62.6 & 22.5 & 62.5 & 17.7 & 58.8 & 90.8 \\
III mean & -0.935 & 5.0 & 2.4 & 2.4 & 3.0 & 3.0 & 3.0 & 2.4 \\
(S.D.) & $(0.009)$ & $(3.5)$ & $(0.8)$ & $(0.8)$ & $(1.3)$ & $(1.3)$ & $(1.3)$ & $(0.8)$ \\
\% var & 0.580 & 16.1 & 14.3 & 14.3 & 14.1 & 14.1 & 14.1 & 14.3 \\
\hline
\end{tabular}

See Table 1 for muscle abbreviations.
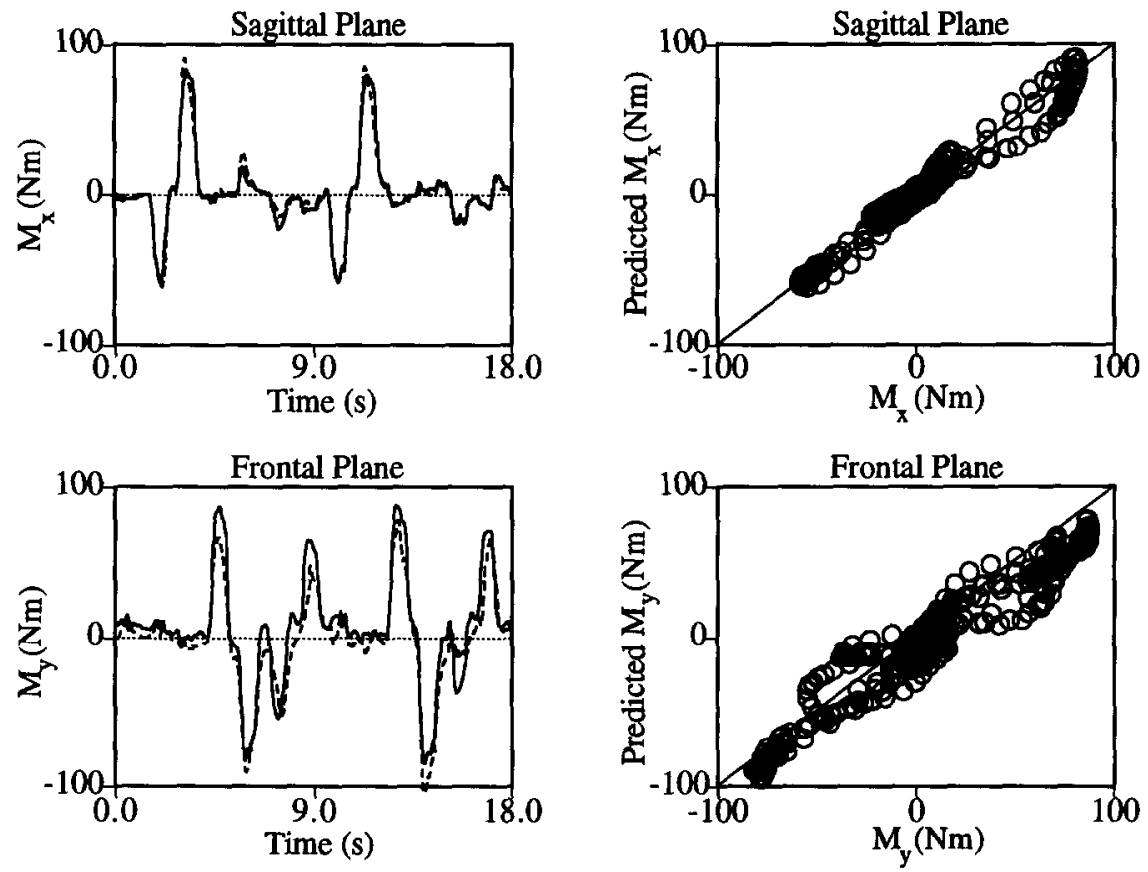

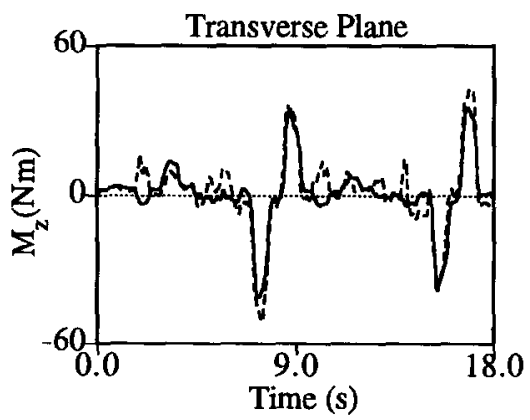

(a)

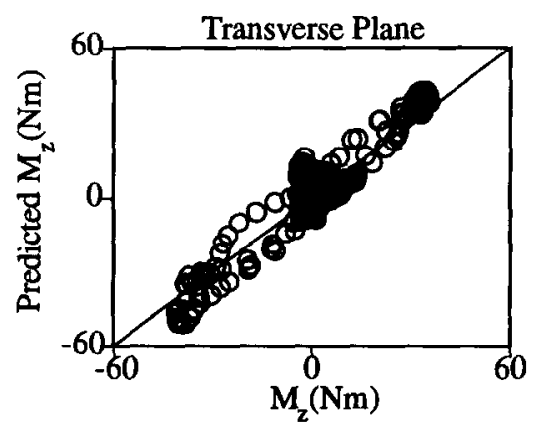

(b)

Fig. 3. (a) Time histories of the measured (solid line) and MES-predicted (broken line) moments during a calibration task. (b) MES-predicted vs measured moment plots demonstrate no systematic bias in the moment predictions. Candidate model III was used to estimate the second-order MES-stress models with no pure time delay. 
Table 4. The estimated second-order RMS MES-stress models, equivalent continuous time constants and dispersion percentages obtained using Model III. The magnitude of the dispersion percentages of the second modes suggests that a second-order model is warranted

\begin{tabular}{|c|c|c|c|c|c|c|c|}
\hline \multirow[b]{2}{*}{ Subject } & \multirow{2}{*}{$\begin{array}{c}\text { Mean (S.D.) gain } \\
b_{i}\end{array}$} & \multicolumn{2}{|c|}{ Characteristic polynomial } & \multicolumn{2}{|c|}{ Time constants } & \multicolumn{2}{|c|}{ Dispersion percentages } \\
\hline & & $a_{1}$ & $a_{2}$ & $\tau_{1}(\mathrm{~ms})$ & $\tau_{2}(\mathrm{~ms})$ & Mode 1 & Mode 2 \\
\hline 1 & $0.65(0.10)$ & -1.72 & 0.74 & 87 & 55 & 274 & -174 \\
\hline 2 & $1.42(1.14)$ & -1.64 & 0.66 & 111 & 31 & 139 & -39 \\
\hline 3 & $0.64(0.25)$ & -1.69 & 0.71 & 99 & 41 & 169 & -69 \\
\hline 4 & $1.32(0.38)$ & -1.57 & 0.59 & 177 & 21 & 113 & -13 \\
\hline 5 & $0.34(0.11)$ & -1.76 & 0.77 & 116 & 58 & 199 & -99 \\
\hline 6 & $1.01(0.33)$ & -1.66 & 0.68 & 114 & 33 & 140 & -40 \\
\hline 7 & $1.04(0.12)$ & -1.53 & 0.55 & 139 & 19 & 115 & -15 \\
\hline 8 & $2.22(0.32)$ & -1.42 & 0.45 & 137 & 14 & 110 & -10 \\
\hline 9 & $0.69(0.49)$ & -1.80 & 0.81 & $*$ & $*$ & 50 & 50 \\
\hline
\end{tabular}

* Estimated RMS MES-stress transfer function is underdamped.

Table 5. Mean (S.D.) maximal exertion muscle stresses and associated contraction forces estimated using the second-order models. Maximal stresses are equal to the DC gain of the RMS MES-stress transfer function and are given by the ratio $b_{i} / A(1)$

\begin{tabular}{lccccccc}
\hline & RA & IO & EO & ESM & ESL & ESI & LD \\
\hline Stress $\left(\mathrm{N} \mathrm{cm}^{-2}\right)$ & $73(46)$ & $38(12)$ & $38(12)$ & $43(19)$ & $43(19)$ & $43(19)$ & $38(12)$ \\
Force (N) & $482(297)$ & $498(164)$ & $623(204)$ & $225(99)$ & $446(196)$ & $431(190)$ & $163(54)$ \\
\hline
\end{tabular}

See Table 1 for muscle abbreviations.
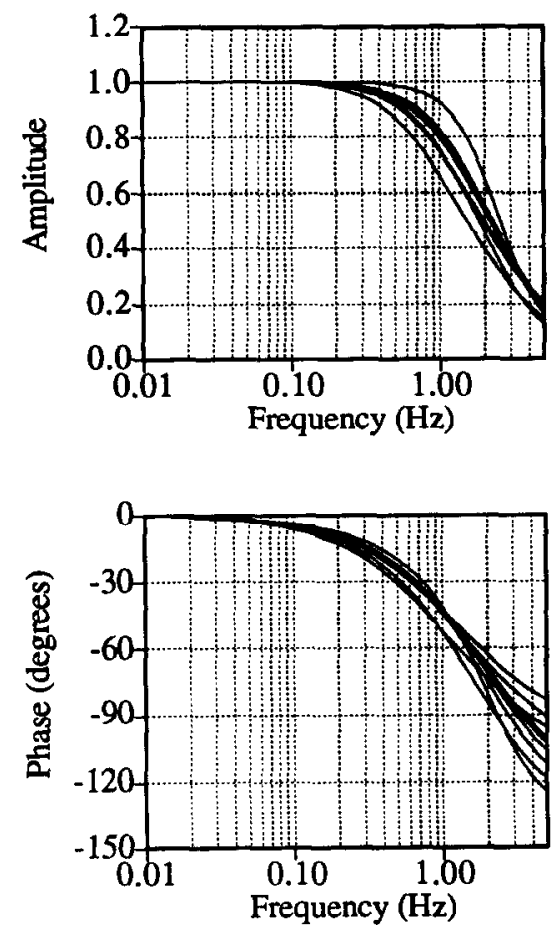

Fig. 4. Normalized frequency responses of the estimated second-order RMS MES-muscle stress transfer functions. The one-half power cutoff frequencies ranged from 0.9 to $1.8 \mathrm{~Hz}$.

all muscles. Accounting for inter-muscle variations in muscle dynamics would involve increased computational complexity and it would need to be substantiated that the MES-force dynamics of different muscles can be separately identified.
An alternative to estimating MES-stress models for individual subjects is to assume some average models can be used to represent the MES-stress relationship of all subjects. McGill and Norman (1986) used such an approach to estimate lumbar muscle forces during dynamic sagittal lifting tasks. In that model, rectified and filtered MES were normalized to maximal activity levels, amplified by an assumed maximum stress level of $35-55 \mathrm{~N} \mathrm{~cm}^{-2}$ and subsequently modulated by muscle length and velocity effects. A time-varying corrective gain factor was then introduced to force MES-predicted and measured sagittal moments to be equal at each time step. This approach assumes that there is an equal probability of error in all muscle force estimates and that adjusting the corrective gain spreads the error equally over all the muscles. Errors in the muscle force estimates can result from many sources including simplified muscle geometry and MES-force modeling, MES crosstalk and inherent variabilities in MES measurements. These errors can become fairly large and systematic during lateral bending and axial twist loadings due to the substantial use of deep trunk and abdominal oblique muscles which are difficult to record MES from and have complex LOA. As an example of such difficulties, McGill (1991) underpredicted an attempted axial twist moment from MES by $84 \%$. Introducing a corrective gain in this case would mask sizable systematic modeling and measurement errors, and result in unrealistically high muscle force estimates.

Marras and Sommerich (1991) and Hughes (1991) have estimated static lumbar MES-stress models from experimental data. Marras and Sommerich normalized MES to maximal activity levels and assumed 

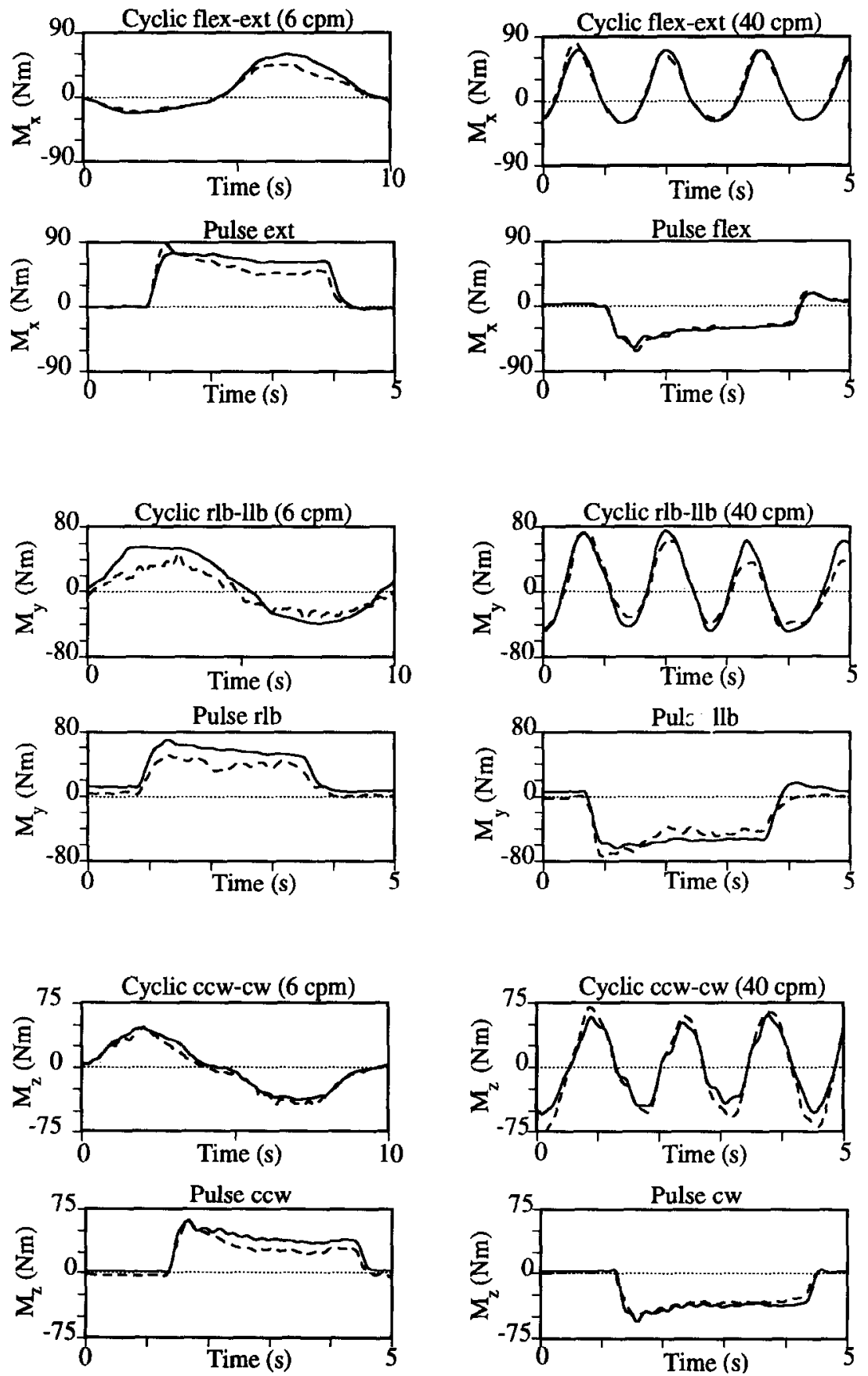

Fig. 5. Sample plots of the measured (solid line) and MES-predicted (broken line) moments during validation tasks involving attempted flexion-extension, attempted lateral bending and attempted axial twist. MES-predicted and measured primary moments were highly correlated in all tasks. However, the magnitudes of the difference between moment traces (moment prediction errors) were largely dependent on the exertion direction.

the MES-stress gains were equal for all muscles included in the model. The MES-stress gain was then adjusted such that model-predicted and measured sagittal moments were in good agreement throughout the performance of isokinetic extension efforts. A shortcoming of that model compared with the present model was that only sagittal plane moments were considered in the parameter estimation despite the performance of some nonsymmetric tasks. Hughes (1991) utilized least-squares estimation to calibrate models relating six lumbar MES and moments in the sagittal and frontal planes. In that model, principal components regression was used to reduce the ill effects of multicolinearity in estimation, and physically feasible and repeatable static models were obtained. In contrast to the results of Hughes, infeasible 
Table 6. Errors in MES-predicted moments, expressed as the mean coefficient of variability, for each of the 12 validation tasks. Moments prediction errors were dependent on the exertion direction but were relatively independent of the rate of exertion

\begin{tabular}{|c|c|c|c|c|c|c|}
\hline \multirow[b]{3}{*}{ Attempted moment } & \multicolumn{6}{|c|}{ Coefficient of variability } \\
\hline & \multicolumn{2}{|c|}{ Sagittal moment } & \multicolumn{2}{|c|}{ Frontal moment } & \multicolumn{2}{|c|}{ Transverse moment } \\
\hline & Mean & (S.D.) & Mean & (S.D.) & Mean & (S.D.) \\
\hline Cyclic flex-ext $(6 \mathrm{cpm})$ & 0.31 & $(0.09)$ & 0.20 & $(0.12)$ & 0.10 & $(0.03)$ \\
\hline Cyclic flex-ext $(40 \mathrm{cpm})$ & 0.29 & $(0.15)$ & 0.22 & $(0.08)$ & 0.12 & $(0.07)$ \\
\hline Pulse flex & 0.29 & $(0.16)$ & 0.25 & $(0.07)$ & 0.10 & $(0.05)$ \\
\hline Pulse ext & 0.21 & $(0.05)$ & 0.13 & $(0.06)$ & 0.08 & $(0.05)$ \\
\hline Cyclic rlb-llb (6 cpm) & 0.26 & $(0.10)$ & 0.34 & $(0.13)$ & 0.16 & $(0.07)$ \\
\hline Cyclic rlb-llb $(40 \mathrm{cpm})$ & 0.25 & $(0.11)$ & 0.33 & $(0.09)$ & 0.21 & $(0.05)$ \\
\hline Pulse rlb & 0.23 & $(0.17)$ & 0.31 & $(0.11)$ & 0.20 & $(0.08)$ \\
\hline Pulse llb & 0.25 & $(0.13)$ & 0.30 & $(0.07)$ & 0.16 & $(0.07)$ \\
\hline Cyclic ccw-cw (6 cpm) & 0.30 & $(0.15)$ & 0.48 & $(0.23)$ & 0.42 & $(0.21)$ \\
\hline Cyclic ccw-cw (40 cpm) & 0.35 & $(0.23)$ & 0.65 & $(0.24)$ & 0.44 & $(0.18)$ \\
\hline Pulse ccw & 0.25 & $(0.13)$ & 0.44 & $(0.23)$ & 0.42 & $(0.20)$ \\
\hline Pulse cw & 0.27 & $(0.15)$ & 0.52 & $(0.24)$ & 0.37 & $(0.23)$ \\
\hline
\end{tabular}

Notation: flex-flexion, ext-extension, rlb-right lateral bend, llb-left lateral bend, ccw-counter-clockwise axial twist, cw-clockwise axial twist.

models were estimated in this study when using principal components analysis. This result may be attributable to the inclusion of twisting moments as well as a larger number (14) of muscles than was used by Hughes.

In the present study, repeatable and physically feasible MES-stress models were obtained by using a minimal number of $a$ priori assumptions to reduce the multicolinearity of model inputs (Model III). MES-predicted moment errors were minimized while MES-stress transfer functions of muscles, whose activities were most highly correlated, were constrained to be the same. The implicit assumption of this constraint was that the MVE stresses generated by muscles with correlated activities are equal. This assumption does not imply that the MVE stresses must be truly maximal but only that the MVE stresses of grouped muscles are equal. These assumptions substantially reduced parameter variability over calibration task repeated trials, and resulted in strictly positive muscle force predictions and estimated MVE muscle stresses that were within maximal limits often used in the literature (McGill, 1991; Schultz, 1983).

An emphasis was placed on identifying dynamic aspects of the MES-force relationship. The temporal aspects of the MES-force relationship are commonly accounted for by simply low-pass filtering rectified or RMS MES with an a priori selected low-pass filter (e.g. Hughes, 1991; McGill and Norman, 1986). Bobet and Norman (1990) demonstrated that dynamic MES-torque models for the elbow could be estimated from experimental data by using a second-order autoregressive with exogenous input (ARX) model representation. The advantage of such a representation is that linear least squares can be used in the parameter estimation stage. The disadvantage, however, is that statistically inconsistent (asymptotically biased) estimates will be obtained when the residual error sequence is not white and the noise-to-signal ratio of standard deviations is 5\% or greater (Ben Mrad and Fassois, 1991). In this study an OE model, based on the difference between measured and MES-predicted moments, was used as the basis for estimating dynamic MES-stress model parameters. The reason for this selection is that OE models can be estimated accurately without directly dealing with any particular forms of noise dynamics (Ljung, 1987). While parameter estimation involves nonlinear optimization, the solution of this problem is significantly facilitated by the direct calculation of the cost function gradient (see Appendix B).

Second-order low-pass transfer functions with no pure time delay were found to be adequate to represent the dynamic RMS MES-force relationship. The one-half power cutoff frequencies of the estimated models $(0.90-1.83 \mathrm{~Hz})$ are comparable to the cutoff frequencies of the low-pass models used to describe the muscular twitch contraction $(2.4 \mathrm{~Hz}$, MilnerBrown et al., 1973) and estimate ankle and knee joint torques from MES (1.0-2.8 Hz, Olney and Winter, 1985). The inclusion of electromechanical delay (EMD), defined as a pure time delay between the onset of MES and the onset of muscular tension (approximately $30 \mathrm{~ms}$, Komi et al., 1987), did not improve moment predictions from MES. This indicates that EMD may be substantially shorter than previously thought (Corcos et al., 1992).

Validation of MES-driven muscle force prediction models remains problematic. Joint moments can be predicted from MES with quantifiable accuracy be- 
cause inverse dynamics can be used to estimate joint moments from external force and motion data (Hof, 1984). However, muscle forces, which are often of greater interest due to their substantial contribution to joint loadings, cannot be measured and thus MESpredicted muscle forces cannot be directly validated. In the present study, model performance was assessed by quantifying the accuracy of MES-predicted moments over a series of tasks involving a range of slow and rapid triaxial exertions. While comparing measured and MES-predicted moments does not validate the accuracy of the MES-predicted muscle forces, it increases one's confidence in the estimated models and provides important information pertaining to the effect of different loading conditions on model performance. Moment prediction errors in the sagittal and frontal planes (13-34\%) during attempted flexionextension and lateral bending were independent of the rate of exertion, and were comparable to error measures obtained during static asymmetric trunk loadings (Hughes, 1991). Moment prediction errors (37-44\%) during attempted twists were substantially smaller than those reported by McGill (1991) but displayed a high degree of inter-subject variability. This variability may be partially attributable to the simplified IO and EO muscle modeling, and crosstalk in the IO and EO MES.

Moment prediction errors were dependent on whether attempted twist was included in the calibration task. Substantially larger sagittal and frontal plane moment prediction errors were obtained during attempted twists when using the models identified from the calibration task without twist. This result illustrates the importance of calibrating and evaluating three-dimensional biomechanical models over fully three-dimensional tasks.

MES measurements provide a means with which to assess muscle recruitment during physical tasks performances. The interpretation of MES in terms of contraction forces allows for a quantitative assessment of the associated joint loads. The present study demonstrates how a calibration method involving system identification techniques can be used to estimate dynamic MES-driven lumbar muscle force prediction models for individual subjects. This type of approach coupled with a thorough assessment of model performance should improve the accuracy with which muscle forces can be estimated from MES.

Acknowledgements--The support of Public Health Service Grants AG 06621, AG 08808 and NS 20536 in this research is gratefully acknowledged.

\section{REFERENCES}

Ben, M. B. and Fassois, S. D. (1991) Recursive identification of vibrating structures from noise corrupted observations. part 2. Performance evaluation via numerical and laboratory experiments. J. Vib. Acoustics 113, 362-368.

Bobet, J. and Norman, R. W. (1990) Least-squares identification of the dynamic relation between the electromyogram and joint moment. J. Biomechanics 23, 1275-1276.
Bogduk, N. and Macintosh, J. E. (1984) The applied anatomy of the thoracolumbar fascia. Spine 9, 164-170.

Corcos, D. M., Gottlieb, G. L., Latash, M. L., Almeida, G. L. and Agarwal, G. C. (1992) Electromechanical delay: an experimental artifact. J. Electromyography Kinesiology 2, 59-68.

Dumas, G. A., Poulin, M. J., Roy, B., Gagnon, M. and Jovanovic, M. (1988) A three-dimensional digitization method to measure trunk muscle lines of action. Spine 13, 532-541.

Fassois, S. D. and Lee, J. E. (1993) On the problem of stochastic experimental modal analysis based on multipleexcitation multiple-response data. Part I. Dispersion analysis of continuous-time structural systems. J. Sound Vib. $161,33-56$.

Hof, A. L. (1984) EMG and muscle force: an introduction. Human Movement Sciences 3, 119-153.

Hughes, R. E. (1991) Empirical evaluation of optimizationbased lumbar muscle force prediction models. Ph.D. thesis, The University of Michigan, Ann Arbor, MI.

Komi, P. V., Salonen, M., Järvinen, M. and Kokko, O. (1987) In vivo registration of achilles tendon forces in man. I. Methodological development. Int. J. Sports Med. 8 (suppl), 3-8.

Kumar, S. (1988) Moment arms of spinal musculature determined from CT scans. Clinical Biomechanics 3, 137-144.

Ladin, Z., Murthy, K. R. and De Luca, C. J. (1989) Mechanical recruitment of low-back muscles: theoretical predictions and experimental validation, Spine 14, 927-938.

Ljung, L. (1987) System Identification: Theory for the User. Prentice-Hall, Englewood Cliffs, New Jersey.

McGill, S. M. (1991) Electromyographic activity of the abdominal and low back musculature during the generation of isometric and dynamic axial trunk torque: implications for lumbar mechanics. J. orthop. Res. 9, 91-103.

McGill, S. M. and Norman, R. W. (1986) Partitioning of the L4-L5 dynamic moment into disc, ligamentous and muscular components during lifting. Spine 11, 666-678.

Macintosh, J. E. and Bogduk, N. (1986) The biomechanics of the lumbar multifidus, Clin. biomech. 1, 205-213.

Marras, W. S. and Sommerich, C. M. (1991) A three-dimensional motion model of loads on the lumbar spine. I. Model structure. Human Factors 33, 123-137.

Milner-Brown, H. S., Stein, R. B. and Yemm, R. (1973) The contractile properties of human motor units during voluntary isometric contractions. $J$. Physiol. 228, 285-306.

McNeil, T., Warwick, D., Andersson, G. and Schultz, A. (1980) Trunk strength in attempted flexion, extension and lateral bending in healthy subjects and patients with low back disorders. Spine 5, 529-538.

Neter, J., Wasserman, W. and Kutner, M. H. (1990) Applied Linear Statistical Methods. Irwin, Illinois.

Olney, S. J. and Winter, D. A. (1985) Predictions of knee and ankle moments of force in walking from EMG and kinematic data. J. Biomechanics 18, 9-20.

Pope, M. H., Andersson, G. B. J., Broman, H., Svensson, M. and Zetterberg, C. (1986) Electromyographic studies of the lumbar trunk musculature during the development of axial torques. J. orthop. Res. 4, 288-297.

Redfern, M. S. (1988) Electromyographic (EMG) signal processing and biomechanical modeling of lower leg muscles. Ph.D. thesis, The University of Michigan, Ann Arbor, MI. Schultz, A. B. and Andersson, G. B. J. (1981) Analysis of loads on the lumbar spine. Spine 6, 76-82.

Schultz, A., Cromwell, R., Warwick, D. and Andersson, G. (1987) Lumbar trunk muscle use in standing isometric heavy exertions. J. orthop. Res. 5, 320-329.

Schultz, A., Haderspeck, K., Warwick, D. and Portillo, D. (1983) Use of lumbar trunk muscles in isometric performance of mechanically complex standing tasks. J. orthop. Res. 1, 77-91.

Thelen, D. G. (1992) A system identification approach to quantifying lumbar trunk loads. Ph.D. thesis, The University of Michigan, Ann Arbor, MI. 
Thelen, D. G., Schultz, A. B. and Ashton-Miller, J. A. (1992) Quantitative interpretation of lumbar myoelectric signals during rapid cyclic attempted flexions and extensions. $J$. Biomechanics (in press).

Tracy, M. F., Gibson, M. J., Szypryt, E. P., Rutherford, A. and Corlett, E. N. (1989) The geometry of the muscles of the lumbar spine determined by magnetic resonance imaging. Spine 14, 186-193.

Veeger, H. E. J., Van Der Helm, F. C. T., Van Der Woude, L. H. V., Pronk, G. M. and Rozendal, R. H. (1991) Inertial and muscle contraction parameters for musculoskeletal modeling of the shoulder mechanism. J. Biomechanics 24, 615-629.

\section{APPENDIX A: PRINCIPAL COMPONENTS PARAMETER ESTIMATION}

The eigenvalues of the input variance-covariance matrix, $\mathbf{R}$, provide a measure of the input variance explained by each of the principal components. In this study nine principal components were adequate to explain over $95 \%$ of the input variance during the calibration task which included attempted axial twist. The OE model inputs of expression (8) were linearly transformed to the principal components by the matrix $\mathbf{S}$, consisting of the nine eigenvectors associated with the largest eigenvalues, through the following transformation expression:

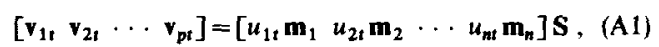

where $n$ is the total number of muscles $(=14)$ and $p$ is the number of principal components used $(=9)$. The relationship between the measured moments and the principal components was written in the OE form:

$$
\mathbf{M}_{\mathbf{t}}=\sum_{i=1}^{p} \frac{c_{i}}{A(B)} \mathbf{v}_{i t-k}+\mathbf{e}_{t}(\mathbf{X})
$$

with $\mathbf{X}$ representing the vector of calibration parameters:

$$
\mathbf{X}=\left[\begin{array}{llllllll}
a_{1} & a_{2} & \cdots & a_{m} & c_{1} & c_{2} & \cdots & c_{p}
\end{array}\right]^{\mathrm{T}} .
$$

After model estimation, the principal component model parameters, $c_{i}$, were related back to the MES-stress gains through the expression:

$$
\left[\begin{array}{llll}
b_{1} & b_{2} & \cdots & b_{n}
\end{array}\right]^{\mathrm{T}}=\mathbf{S}\left[\begin{array}{llll}
c_{1} & c_{2} & \cdots & c_{p}
\end{array}\right]^{\mathrm{T}} .
$$

\section{APPENDIX B: OPTIMIZATION ROUTINE}

The candidate models are of the OE form

$$
\mathbf{M}_{t}=\sum_{i=1}^{n} \frac{g_{i}}{A(B)} \mathbf{x}_{i t-k}+\mathbf{e}_{t}(\mathbf{X})
$$

where $\mathbf{M}_{t}$ is the measured output, $\mathbf{x}_{i t-k}$ is the vector input at time $t-k, n$ is the total number of inputs, $A(B)$ is the characteristic polynomial, $g_{i}$ is the numerator parameter for input $i$, and $\mathbf{e}_{t}(\mathbf{X})$ is the output error vector at time step $t$. The model parameters, collected in a vector $\mathbf{X}$, are estimated by minimizing a nonlinear cost function $V$ defined as one half the trace of the output error covariance matrix:

$$
\mathbf{V}=\operatorname{Trace}\left[\frac{1}{2 N} \sum_{i=1}^{N} \mathbf{e}_{i}(\mathbf{X}) \mathbf{e}_{i}^{\mathrm{T}}(\mathbf{X})\right]
$$

The nonlinear optimization problem is solved using an iterative Gauss-Newton type algorithm (Ljung, 1987). The means of all model input and output data sequences are removed prior to estimation, and an initial estimate of the model parameters, $\hat{\mathbf{X}}^{(0)}$, is made. The search routine is then given as:

$$
\hat{\mathbf{X}}^{(i+1)}=\hat{\mathbf{X}}^{(i)}-\mu^{(i)}\left[\mathbf{H}_{\mathbf{N}}\left(\hat{\mathbf{X}}^{(i)}\right)\right]^{-1} \mathbf{V}_{N}^{\prime}\left(\hat{\mathbf{X}}^{(i)}\right),
$$

where $N$ is the number of data points, $\mathbf{V}_{N}^{\prime}\left(\hat{\mathbf{X}}^{(i)}\right)$ is the gradient of the cost function with respect to the parameter vector during iteration $i$, and $\mathbf{H}_{\mathbf{N}}\left(\hat{\mathbf{X}}^{(i)}\right)$ is the estimated Hessian of the cost function given in (B2). The gradients and Hessian are calculated directly through the following expressions:

$$
\begin{aligned}
& \mathbf{V}_{N}^{\prime}(\mathbf{X})=-\frac{1}{N} \sum_{t=1}^{N} \mathbf{G}_{t}(\mathbf{X}) \mathbf{e}_{t}(\mathbf{X}), \\
& \mathbf{H}_{\mathbf{N}}(\mathbf{X})=\frac{1}{N} \sum_{t=1}^{N} \mathbf{G}_{t}(\mathbf{X}) \mathbf{G}_{t}^{\mathrm{T}}(\mathbf{X})
\end{aligned}
$$

where $\mathbf{G}_{i}\left(\hat{\mathbf{X}}^{(j)}\right)$ is the gradient matrix of the output error:

$$
\mathbf{G}_{\mathbf{t}}(\mathbf{X})=\left[\frac{\partial \hat{\mathbf{M}}_{t}(\mathbf{X})}{\partial a_{1}} \cdots \frac{\partial \hat{\mathbf{M}}_{t}(\mathbf{X})}{\partial a_{m}} \frac{\partial \hat{\mathbf{M}}_{t}(\mathbf{X})}{\partial g_{1}} \cdots \frac{\partial \hat{\mathbf{M}}_{t}(\mathbf{X})}{\partial g_{n}}\right]^{\mathbf{T}}
$$

with:

$$
\begin{gathered}
\frac{\partial \hat{\mathbf{M}}_{t}(\mathbf{X})}{\partial a_{j}}=-\sum_{i=1}^{n} \frac{g_{i}}{A(B) A(B)} \mathbf{x}_{i t-k-j}, \\
\frac{\partial \hat{\mathbf{M}}_{t}(\mathbf{X})}{\partial g_{j}}=\frac{1}{A(B)} \mathbf{x}_{j t-k} .
\end{gathered}
$$

At each iteration, the scalar step size $\mu^{(i)}$ is set equal to 1.0, and then bisected up to ten times until a lower value of the cost function is obtained. The iterations are terminated when the search direction vector has a norm less than a specified tolerance limit, or when a lower value of the cost function cannot be found. 